Instructions for authors, subscriptions and further details:

\title{
Burnout y Factores de Resiliencia en Docentes de Educación Secundaria
}

M. Inmaculada Vicente de Vera ${ }^{1}$

M. Inés Gabari Gambarte ${ }^{1}$

1) Universidad Pública de Navarra, Spain

Date of publication: June $25^{\text {th }}, 2019$

Edition period: June 2019-October 2019

To cite this article: Vicente de Vera, M.I. \& Gabari Gambarte, M.I. (2019). Burnout y Factores de Resiliencia en Docentes de Educación Secundaria, International Journal of Sociology of Education, 8(2), 127-152. doi: $10.17583 /$ rise.2019.3987

To link this article: http://dx.doi.org/10.17583/rise.2019.3987

\section{PLEASE SCROLL DOWN FOR ARTICLE}

The terms and conditions of use are related to the Open Journal System and to Creative Commons Attribution License (CC-BY) 


\title{
Burnout and Resilience Factors in High School Teachers
}

M. Inmaculada Vicente de Vera

Universidad Pública de Navarra
M. Inés Gabari Gambarte

Universidad Pública de Navarra

(Received: 4 January 2019; Accepted: 13 May 2019; Published: 25 June 2019)

\begin{abstract}
The teacher's task imposes physical, mental, emotional and relational demands that, in interaction with individual resources, can negatively impact on their health and well-being. Resilience in education is the ability to resist, it is the exercise of strength to face all the vicissitudes of personal, family, professional and social life. Through empirical study, relationships are established between the perceptions of burnout and resilience measured in the participants. The invited sample is composed of Secondary Education teachers $(\mathrm{N}=1.268)$ of the twenty-four Institutes of the province of Huesca (Spain) belonging to the public education network in the 2014/15 academic year. Through voluntary participation, the final sample is formed with $n=167$ teachers $(13.17 \%)$. For the measurement of burnout, the adaptation to Spanish (MBI-GS) of Salanova, Schaufeli, Llorens, Peiró and Grau (2000) is used. Resilience is evaluated through the Resilience Evaluation Questionnaire of Serrano Martínez (2010). The results identify $1.20 \%$ subjects with burnout and $13.17 \%$ with resilience. It is concluded that resilience decreases vulnerability to burnout. Variables such as positivism, constructive coping strategies, tolerance to frustration, the locus of internal control and self-efficacy are identified as protection factors against the syndrome.
\end{abstract}

Keywords: burnout; resilience; self-efficacy; positivism; coping strategies; control locus. 


\section{Burnout y Factores de Resiliencia en Docentes de Educación Secundaria}

M. Inmaculada Vicente de Vera

Universidad Pública de Navarra
M. Inés Gabari Gambarte

Universidad Pública de Navarra

(Recibido: 4 Enero 2019; Aceptado: 13 Mayo 2019; Publicado: 25 Junio 2019)

\section{Resumen}

La tarea del docente impone demandas físicas, mentales, emocionales y relacionales que, en interacción con los recursos individuales, pueden impactar negativamente en su salud y bienestar. La resiliencia en educación es la capacidad de resistir, es el ejercicio de la fortaleza para afrontar todos los avatares de la vida personal, familiar, profesional y social. Mediante estudio empírico, se establecen relaciones entre las percepciones de burnout y resiliencia medidos en los participantes. La muestra invitada está compuesta por profesorado de Educación Secundaria $(\mathrm{N}=1.268)$ de los veinticuatro Institutos de la provincia de Huesca (España) pertenecientes a la red pública de enseñanza en el curso 2014/15. Mediante participación voluntaria, se conforma la muestra definitiva con $\mathrm{n}=167$ docentes $(13.17 \%)$. Para la medida del burnout se emplea la adaptación al castellano del (MBI-GS) de Salanova, Schaufeli, Llorens, Peiró y Grau (2000). La resiliencia se evalúa mediante el Cuestionario de Evaluación de Resiliencia de Serrano Martínez (2010). Los resultados identifican $1,20 \%$ sujetos con burnout y $13.17 \%$ con resiliencia. Se concluye que la resiliencia disminuye la vulnerabilidad al burnout. Se identifican variables como el positivismo, las estrategias de afrontamiento constructivas, la tolerancia a la frustración, el locus de control interno y la autoeficacia, como factores de protección frente al síndrome.

Palabras clave: burnout; resiliencia; autoeficacia; positivismo; estrategias de afrontamiento; locus de control. 


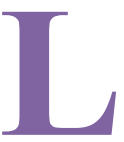

os estudios del burnout en los profesionales de la educación evidencian que la disminución de los recursos dedicados a la enseñanza y los cambios curriculares (Longas, Chamarro, Riera y Cladellas, 2012), la sobrecarga de trabajo con exceso de tareas (Rodríguez, Sola y Fernández, 2017), las presiones temporales (López y Extremera, 2017), los conflictos interpersonales (Justo, 2010; Carlotto y Câmara, 2017), el escaso grado de autonomía (Burgos, Paris, Salcedo y Arriagada, 2018) y la falta de salud organizacional (Llull, Cerdà y Brage, 2015; Vicente de Vera y Gabari, 2019), entre otros factores, están relacionadas con el cansancio emocional de los docentes.

Sin embargo, a pesar de que los eventos organizacionales pueden ser por sí mismos suficientemente estresantes, algunos sujetos son más vulnerables que otros a desarrollar el síndrome (Albar et al., 2004). Ello depende, en parte, de las estrategias cognitivas que se tengan para resolver los problemas, del tipo de ejercicio profesional y del desarrollo de factores de resistencia o de protección que se encuentran ligados a las variables individuales, como el tipo de personalidad y atributos o rasgos de la misma (Vargas, Niño y Acosta, 2017).

Existen algunos rasgos individuales (como la resiliencia, autoeficacia, resistencia a la frustración, locus de control, expectativas, etc.), que ejercen una gran influencia en la evaluación que las personas hacen de las situaciones estresantes en función de los rasgos cognitivos de la personalidad que, a su vez, condicionan el tipo de afrontamiento para responder a dicha situación estresante (González, Torres y Carreres, 2017; Noreña, 2018).

La resiliencia es entendida como una fortaleza que el ser humano desarrolla ante la adversidad, que le permite resistir el suceso estresante, rehacerse del mismo, e incluso salir fortalecido de la experiencia (Bonanno, 2004). Numerosos estudios corroboran que la resiliencia disminuye la vulnerabilidad al burnout (Stratta et al., 2013; Ornelas, 2016; Cruz y Puentes, 2017), siendo la capacidad de afrontamiento a los estresores una de las claves de la resiliencia. De modo que, el uso de estrategias de afrontamiento constructivas centradas en el problema (como la búsqueda de apoyo social instrumental, la reinterpretación positiva y la aceptación) previene el desarrollo del síndrome (Medrano, 2017; Alarcón, 2018). Mientras que el uso de estrategias de afrontamiento pasivas centradas en la emoción (desahogo, negación y búsqueda de apoyo social emocional) 
facilita su aparición (Aldás, 2017; Félix Verduzco, García Hernández y Mercado Ibarra, 2018). El burnout aparece cuando las estrategias de afrontamiento están más centradas en la emoción y la evitación.

Adicionalmente, algunas investigaciones sugieren que las emociones positivas deshacen los efectos fisiológicos que provocan las emociones negativas. Subrayan, el peso que emociones positivas como el optimismo, asertividad y la autoestima, entre otros aspectos, tienen en la construcción del proceso resiliente (McKergow, 2009). Se ha encontrado que una actitud optimista ante situaciones retadoras se relaciona con la confianza y la persistencia en la conducta, aunque las cosas se pongan difíciles (Oriol, Mendoza, Covarrubias y Molina, 2017). Por el contrario, una actitud pesimista hace que las personas se manifiesten dudosas y vacilantes (Pulido y Herrera, 2018).

Otra de las variables con más relevancia dentro de la personalidad positiva que guarda cierta similitud con el concepto de resiliencia, es la fortaleza de carácter o también llamada personalidad resistente. Las personas con este tipo de personalidad padecen menos estrés laboral y tienden a desgastarse menos profesionalmente (González, Pelegrín y Carballo, 2017). Todo esto lleva a que, si se produce un acontecimiento estresante, el individuo tiene más estrategias para amortiguarlo y no verlo solo en el sentido negativo, sino como un reto (Vargas et al., 2017; Carrara, 2018).

Asimismo, recientes estudios aseveran que la resiliencia mejora o mantiene, entre otros factores, la eficacia profesional (Tejedor y Mangas, 2016). Por una parte, porque las personas autoeficaces no suelen percibir las demandas ambientales como estresantes $\mathrm{y}$, por otra, porque se esfuerzan más (Vélez, López y González, 2017). De hecho, numerosos trabajos defienden que el burnout se produce como consecuencia de crisis sucesivas de eficacia (Vallejo, 2017; Dios, Calmaestra y Rodríguez, 2018). Estas creencias han mostrado su potencial predictivo en el desarrollo del síndrome. Los mencionados autores explican que un sujeto implicado, con altos niveles de control y preparado para afrontar retos, que utiliza las estrategias de afrontamiento adecuadas, realiza su trabajo de forma más eficaz sin rendirse ante las tareas exigentes. Por tanto, la falta de autoconfianza en realizar bien el trabajo aumenta la probabilidad de sufrir burnout (Lozano y Reyes, 2017; Peña, Raso y Ferrero, 2018). 
Otra de las variables personales que la literatura científica asocia al desarrollo del síndrome, es el locus de control (Esteras, Sandín y Chorot, 2016). Las personas con locus de control interno tienen la creencia de que los eventos vitales y sus consecuencias son controlados por sus propias decisiones y acciones. En cambio, los sujetos con locus de control externo tienen el convencimiento de que dichos eventos son controlados por fuerzas externas, otras personas, la suerte o el destino (Vargas, Briñez, Segura y Nieto, 2016). Estas creencias son relativamente estables y tienen importantes implicaciones en el rendimiento y en el bienestar en el trabajo. Es decir, las personas con locus de control interno, al percibir que tienen dominio sobre las situaciones, tienen expectativas de afrontamiento más favorables y se enfrentan a los problemas realizando acciones que contrarrestan los efectos de las condiciones adversas (Torres y Bonilla, 2017). Su experiencia, por tanto, resulta menos amenazante ante los estresores que la de las personas con locus de control externo, más propensas al desamparo, la vulnerabilidad y la insatisfacción laboral (Islas, Gutiérrez, Castellanos y Méndez, 2017).

El trabajo que a continuación presentamos, tiene como objetivo principal establecer relaciones entre el burnout percibido y factores de resiliencia en docentes de Educación Secundaria de la provincia de Huesca (España). Como objetivo secundario nos planteamos comprobar los niveles de eficacia percibida en dicho colectivo y su influencia frente al burnout. De modo que, dadas las diferencias individuales en la implementación de las estrategias de afrontamiento ante condiciones amenazantes o estresantes, es plausible pensar que la resiliencia, así como la autoeficacia percibida, pueden ser dos moduladores destacados del bienestar del profesorado de este estudio.

\section{Método}

El diseño de este estudio fue transversal, encuadrándose dentro de un modelo descriptivo y correlacional. La variable independiente fue la pertenencia a un colectivo docente de Enseñanza Secundaria de la provincia de Huesca (España). Las variables dependientes, los niveles de burnout y de resiliencia percibidos. 


\section{Vicente de Vera \& Gabari Gambarte - Burnout y Resiliencia}

\section{Participantes}

La muestra invitada estuvo compuesta por la población de docentes de Educación Secundaria de los veinticuatro Institutos de la provincia de Huesca pertenecientes a la red pública de enseñanza en el curso 2014/15 $(\mathrm{N}=1.268)$. El criterio de muestreo fue la participación voluntaria. La muestra aceptante estuvo conformada por 167 profesores y profesoras, lo que supuso una respuesta del $13,17 \%$.

\section{Instrumentos}

La recogida de información se realizó mediante la modalidad de encuesta en Google Drive, previa solicitud de conformidad con los directores de los centros. El instrumento empleado fue un cuestionario en Google Drive de 55 ítems tipo Likert que combinó otros dos:

1) Para la medida del burnout se escogió la adaptación al castellano del Maslach Burnout Inventory-General Survey (MBI-GS, Schaufeli, Leiter, Maslach y Jackson, 1996) de Salanova, Llorens, Grau, Schaufeli y Peiró (2000). El cuestionario lo conformaron 15 ítems estructurados en una escala tipo Likert, con rango de frecuencia desde nunca/ninguna vez (0) a siempre/todos los días (6). Dichos ítems se distribuyeron en las tres escalas del constructo: agotamiento emocional (5 ítems), cinismo (4 ítems) y eficacia personal/profesional (6 ítems).

2) La resiliencia se evaluó mediante el Cuestionario de Evaluación de Resiliencia de Serrano Martínez (2010), con 34 cuestiones estructuradas en una escala tipo Likert, con cinco niveles de repuesta desde nunca (1) a siempre (5). La evaluación psicométrica del cuestionario evidenció 8 ítems como inconsistentes, por lo que fueron eliminados. El análisis factorial exploratorio obtuvo cinco factores: sensibilidad o afrontamiento emocional (6 ítems), superación o afrontamiento conductual ( 8 ítems), positivismo u optimismo ingenuo (6 ítems), pensamiento estereotipado (4 ítems) y tolerancia a la frustración (4 ítems).

El Factor I, se llama sensibilidad o afrontamiento emocional. La persona no pretende modificar la situación problemática, sino modular los estados emocionales negativos y sobrevivir a ellos (Lázarus y Folkman, 1986). Las estrategias de afrontamiento son el autocontrol, el distanciamiento, la 
revaluación positiva, la autoinculpación y el escape/evitación. El Factor II, se denomina superación o afrontamiento conductual. Mide la tendencia a pensar automáticamente en términos que faciliten una acción eficaz. Los recursos de afrontamiento son todos aquellos pensamientos, reinterpretaciones, conductas, etc., que el individuo puede desarrollar para tratar de conseguir los mejores resultados posibles en una determinada situación (Lázarus y Folkman, 1986). Respecto al Factor III, recoge aspectos que aluden al positivismo u optimismo ingenuo. Este componente se refiere a la capacidad para pensar favorablemente, sabiendo enfatizar el lado positivo de la situación. El Factor IV, se llama pensamiento estereotipado. Es la tendencia de algunas personas a dejarse llevar por las ideas establecidas socialmente. Por último, el Factor V, se denomina tolerancia a la frustración. Mide la capacidad de la persona para afrontar los problemas y limitaciones que se encuentra a lo largo de la vida, a pesar las molestias o incomodidades que estas le causan.

Asimismo, 4 cuestiones recogieron información sobre variables sociodemográficas: sexo, rango de edad, estado civil y número de hijos/as y otras 2 cuestiones, sobre variables sociolaborales del puesto de trabajo del docente: antigüedad, estabilidad (contratado/a, funcionario/a, interino/a o sustituto/a). Se decidió no solicitar la identificación del centro de trabajo para evitar una disminución en el nivel de respuesta, tradicionalmente bajo en estos temas.

\section{Procedimiento}

Se contactó telefónicamente con los equipos directivos de los centros educativos solicitando su colaboración para la difusión del cuestionario entre los docentes mediante correo electrónico. Se diseñó un cuestionario único en Google Drive y se hizo llegar al profesorado invitado asegurando el anonimato, así como explicitando el objetivo de la recogida de datos. Se estableció un plazo de recepción de respuesta de un mes. Se realizó un análisis previo para descartar los cuestionarios incompletos o erróneos. 


\section{Vicente de Vera \& Gabari Gambarte - Burnout y Resiliencia}

\section{Análisis de Datos}

La relación entre las puntuaciones de los factores de la extracción factorial se midió mediante coeficientes de correlación. Con el fin de que los resultados fuesen suficientemente significativos, se tuvieron en cuenta valores de $r$ con una significación estadística de $p=0,01$. Se consideró correlación en valores de $\mathrm{p}>0,20$, ya que la muestra no fue representativa. Para el análisis estadístico descriptivo, correlacional y factorial se empleó el paquete estadístico SPSS versión 20. La estructura factorial de las escalas se comprobó mediante análisis factorial exploratorio con Rotación de tipo Varimax. Previo a la realización del análisis factorial se confirmó su adecuación con el índice KMO y el test de Barlett.

\section{Resultados}

La distribución de la muestra fue: 94 mujeres $(56,29 \%)$ y 73 varones $(43,71 \%)$. El mayor porcentaje acumulado se situó entre los 46 y los 55 años $(38,92 \%)$, por lo que nos encontramos en una franja de edad en la que habitualmente emergen los casos de burnout. Predominó el profesorado con una experiencia de ejercicio docente de más de veinte años (44,91\%), aspecto que pudo actuar como factor protector dada la seguridad que aporta la experiencia laboral, pero que también pudiera tener repercusiones negativas en cuanto a pérdida de sintonía. Un alto porcentaje $(70,66 \%)$ fueron funcionarios/as, estabilidad laboral que posiblemente actuó como factor de protección, pero que, a su vez, se encuadra en un ámbito laboral donde surgen más problemas relacionados con el agotamiento. E1 73,65\% de los docentes que colaboraron en este estudio estaban casados/as o viviendo en pareja, lo que también se identificó como posible factor de protección en términos de resiliencia. En lo que se refiere a la descendencia, destacó la participación del profesorado con hijos/as. El 66,47\% del profesorado tenía algún hijo/a. De nuevo se encontró una particularidad que pudiera actuar como factor de protección. 
Tabla 1

Distribución sociodemográfica de la muestra aceptante.

\begin{tabular}{lcccc}
\hline & Hombre & Mujer & Total & Porcentaje \\
\hline Entre 22 y 35 años & 6 & 15 & 21 & 12,57 \\
Entre 36 y 45 años & 22 & 32 & 54 & 32,34 \\
Entre 46 y 55 años & 31 & 34 & 65 & 38,92 \\
Entre 56 y 65 años & 14 & 13 & 27 & 16,17 \\
\hline Menos de tres años de experiencia & 4 & 2 & 6 & 3,59 \\
De tres a cinco años de experiencia & 6 & 5 & 11 & 6,59 \\
De cinco a quince años de experiencia & 13 & 35 & 48 & 28,74 \\
Entre quince y veinte años de experiencia & 14 & 13 & 27 & 16,17 \\
Más de veinte años de experiencia & 39 & 36 & 75 & 44,91 \\
\hline Funcionario & 52 & 66 & 118 & 70,66 \\
Contratado & 8 & 10 & 18 & 10,78 \\
Interino & 12 & 16 & 28 & 16,77 \\
Sustituto & 1 & 2 & 3 & 1,79 \\
\hline Casado/a o viviendo en pareja & 54 & 69 & 123 & 73,65 \\
Soltero/a & 11 & 10 & 21 & 12,57 \\
Otras situaciones & 2 & 5 & 7 & 4,20 \\
No indican estado civil & 6 & 10 & 16 & 9,58 \\
\hline Ningún hijo/a & 22 & 34 & 56 & 33,53 \\
Un hijo/a & 15 & 22 & 37 & 22,15 \\
Dos hijos/as & 28 & 32 & 60 & 35,93 \\
Tres hijos/as & 7 & 5 & 12 & 7,19 \\
Más de tres hijos/as & 1 & 1 & 2 & 1,20 \\
\hline Total & 73 & 94 & 167 & 100,0 \\
\hline
\end{tabular}

La corrección del cuestionario del burnout se realizó conforme establece el manual de Bresó, Salanova, Schaufeli y Nogareda (2007), de modo que los valores de los factores (agotamiento, cinismo y eficacia personal/profesional), fueron un valor medio. Se repitió esta operación para cada una de las escalas y para cada uno de los sujetos a los que les fue administrado el instrumento de medida.

Con el fin de saber qué porcentaje de profesorado sufría el síndrome, se corrigieron las respuestas del cuestionario del burnout mediante percentiles. En este sentido, el manual del MBI incluye a los sujetos por encima del 


\section{Vicente de Vera \& Gabari Gambarte - Burnout y Resiliencia}

percentil 75 en la categoría alto índice de burnout, entre el percentil 25 y el 75 en la categoría medio y por debajo del percentil 25 en la categoría bajo índice de burnout. Así, el profesorado de este estudio manifestó bajos índices de burnout. En concreto, se apreciaron índices positivos en burnout por debajo del percentil 25. En cambio, por encima del percentil $25(n=46)$ se obtuvieron valores positivos en eficacia.

Tras el análisis de datos se identificaron con nitidez 2 sujetos con burnout (1,20\% de la muestra), diagnosticados con agotamiento y cinismo elevados y eficacia baja; también aparecieron 7 casos $(4,19 \%$ de la muestra) en los que se hallaron agotamiento y cinismo elevados con eficacia normal, que nos hicieron sospechar que pudiese haber más casos (ver Tabla 2).

Tabla 2

Evaluación de burnout en docentes de Secundaria de Huesca (España) curso 2014/15

\begin{tabular}{lccc} 
Casos burnout & $\mathrm{n}$ & Porcentaje & $\begin{array}{c}\text { Índice de } \\
\text { burnout }\end{array}$ \\
\hline Síndrome de burnout & 2 & 1,20 & Alto \\
\hline Sin burnout con eficacia $<4$ & 6 & 3,59 & Bajo \\
\hline Sin burnout con agotamiento $>5$ & 16 & 9,58 & Medio \\
\hline Sin burnout con cinismo $>5$ & 9 & 5,39 & Medio \\
\hline $\begin{array}{l}\text { Sin burnout } \text { con agotamiento }>5 \text { y } \\
\text { cinismo }>5\end{array}$ & 7 & 4,19 & Medio \\
\hline
\end{tabular}

El primero de los sujetos con burnout fue un varón cuyo rango de edad está entre 46 a 55 años, de estado civil no identificable (por la respuesta en blanco), sin hijos/as y funcionario con 15 a 20 años de experiencia docente. El segundo caso fue una mujer, con edad entre los 46 a 55 años, separada y con un hijo. Era funcionaria y contaba con 15 a 20 años de experiencia docente. Cabe señalar, que ambos sujetos tenían edad madura, larga trayectoria laboral, 'seguridad' en su puesto de trabajo, al que habían accedido por oposición; en cambio, les diferenció su sexo y su composición de unidad familiar. Es decir, mostraron más incidencia en el síndrome aquellas características relacionadas con el entorno laboral, frente a las que 
actuaron como factores de protección, en relación con los soportes sociales. La Figura 1 muestra una comparativa de las medias obtenidas en cada una de las dimensiones de la escala del burnout.

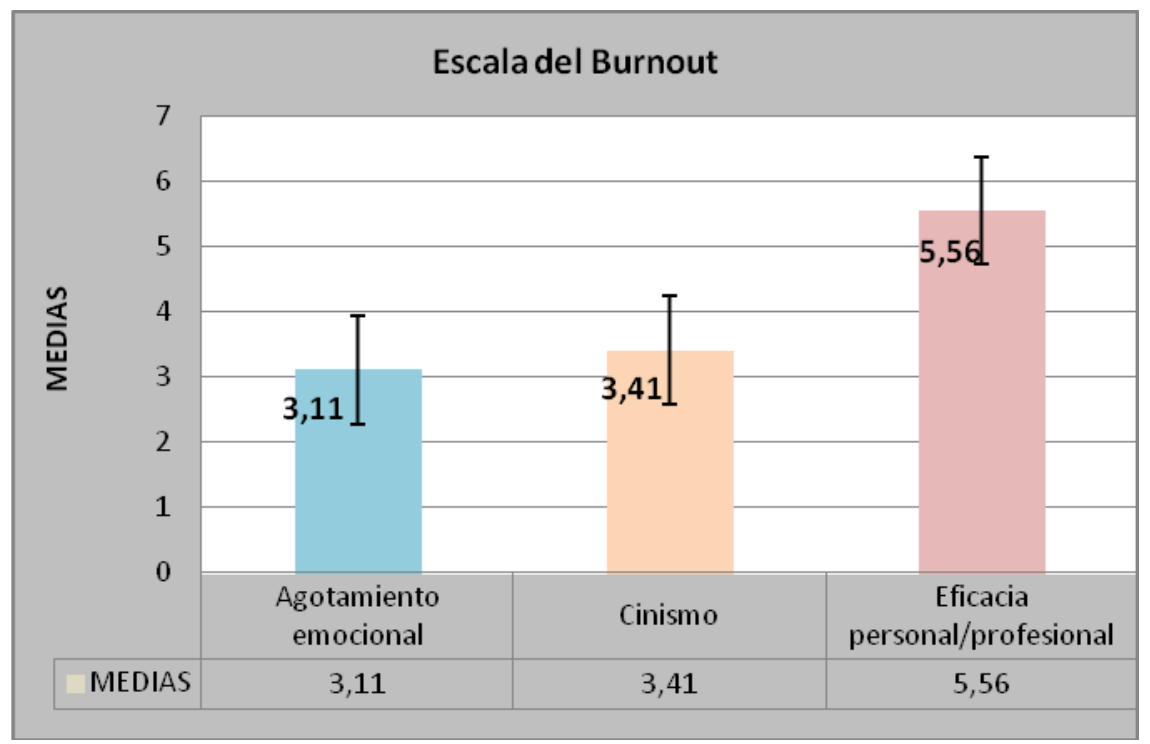

Figura 1. Medias de los factores de la escala del Burnout

La corrección del cuestionario de resiliencia se realizó hallando para cada uno de los cinco factores de la escala, primero el sumatorio y después la media de las preguntas que lo componen. Para saber qué porcentaje de la muestra era resiliente, se corrigieron también las respuestas de dicho cuestionario mediante percentiles. Para ello, se empleó la mediana, sabiendo que corresponde con el percentil 50. Esto indicó que existía una tendencia a considerar a los docentes que superasen dicha mediana como posibles resilientes. Tras el análisis, el grupo de docentes participantes se situó entre el percentil 25 y el 75, en la categoría medio índice de resiliencia. 


\section{Vicente de Vera \& Gabari Gambarte - Burnout y Resiliencia}

Tabla 3

Evaluación de resiliencia en docentes de Secundaria de Huesca (España) curso 2014/15

Factores de resiliencia Participantes que superan la mediana en cada factor

\begin{tabular}{lc}
$\begin{array}{l}\text { Factor I: sensibilidad o afrontamiento } \\
\text { emocional }\end{array}$ & $91(54,49 \%)$ \\
$\begin{array}{l}\text { Factor II: superación o afrontamiento } \\
\text { conductual }\end{array}$ & $88(52,69 \%)$ \\
$\begin{array}{l}\text { Factor III: positivismo u optimismo } \\
\text { ingenuo }\end{array}$ & $90(53,89 \%)$ \\
Factor IV: pensamiento estereotipado & \\
Factor V: tolerancia a la frustración & $84(50,30 \%)$ \\
\hline
\end{tabular}

Un total de 22 participantes (13.17\%), de ellos 13 mujeres y 9 hombres, superaron la mediana de los cinco factores a la vez y fueron considerados resilientes. Según estos datos, parece que la resiliencia tendió a desarrollarse más en el colectivo de docentes mujeres que en el de hombres. De los 22 sujetos hallados resilientes, 19 estaban casados/as o viviendo en pareja, 2 eran solteros/as y uno/a no indicó su estado civil. Además, 14 de ellos/as tenían hijos/as. Respecto a la edad, 9 de ellos/as se encontraban entre los 46 a 55 años; 6 sujetos se situaban en la franja de 56 a 65 años; 4 en la franja de 36 a 45 años y, por último, otros 2 sujetos tenían edades que oscilaban entre $\operatorname{los} 22$ a 35 años.

De estos 22 sujetos hallados resilientes 17 eran funcionarios/as y 12 de ellos/as contaban con una experiencia profesional de más de 20 años de ejercicio docente. En la Figura 2, se expone una representación de las medias obtenidas en cada uno de los factores de la escala de la resiliencia. 


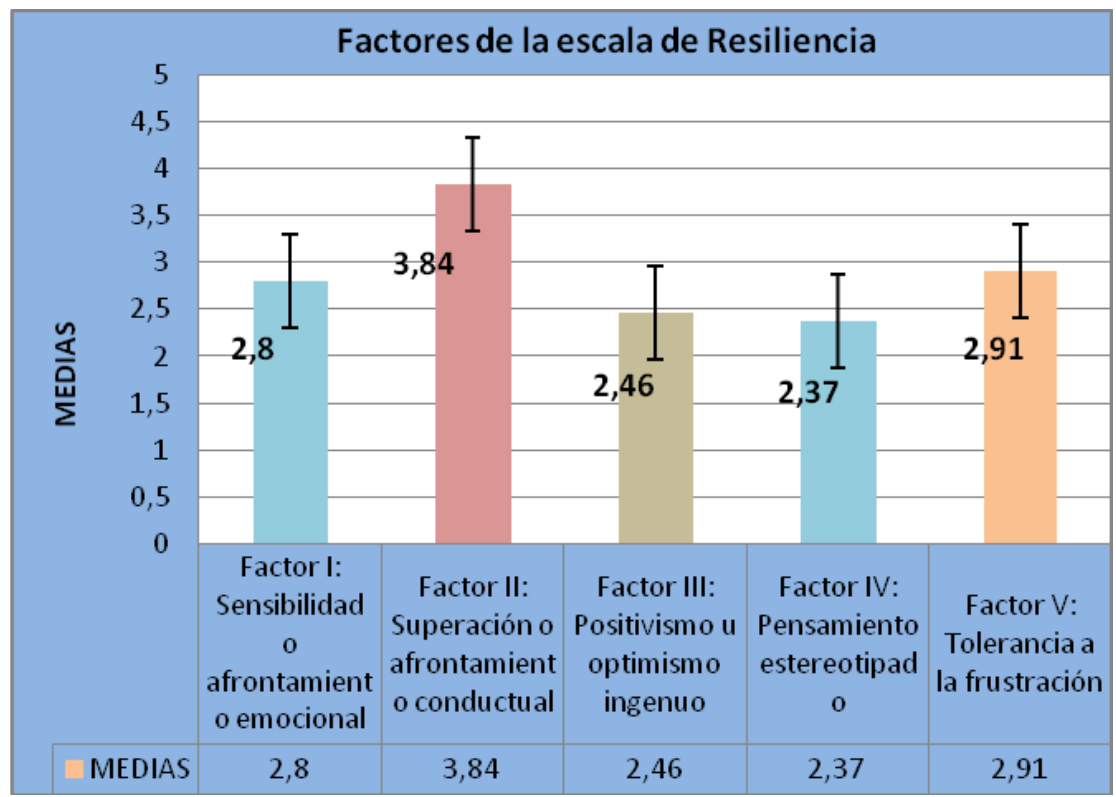

Figura 2. Medias de los factores de la escala de Resiliencia

Por otro lado, en la Tabla 4 se presenta el estudio correlacional de los cuestionarios de burnout y de resiliencia.

Tabla 4

Correlaciones de los factores del Burnout con los factores de la Resiliencia.

\begin{tabular}{|c|c|c|c|c|c|c|c|c|}
\hline & Agota. & Cinismo & Eficacia & Factor I & Factor II & Factor III & Factor IV & Factor V \\
\hline Agotamiento & 1 & $0,58 * *$ & $-0,24 * *$ & $0,37 * *$ & $0,20 * *$ & $0,33 * *$ & $0,61 * *$ & $0,58 * *$ \\
\hline Cinismo & $0,58 * *$ & 1 & $-0,21 * *$ & $0,30 * *$ & 0,06 & $0,29 * *$ & $0,47^{* *}$ & $0,48 * *$ \\
\hline Eficacia & $-0,24 * *$ & $-0,21^{* *}$ & 1 & $-0,18^{*}$ & $0,31 * *$ & $-0,34^{* *}$ & $-0,41 * *$ & $-0,31^{* *}$ \\
\hline Factor I & $0,37^{* *}$ & $0,30^{* *}$ & $-0,18^{*}$ & 1 & $0,32 * *$ & $0,56^{* *}$ & $0,43^{* *}$ & $0,50 * *$ \\
\hline Factor II & $-0,20 * *$ & $-0,06$ & $0,31^{* *}$ & $0,32 * *$ & 1 & $0,19^{*}$ & 0,08 & $0,26^{* *}$ \\
\hline Factor III & $-0,33 * *$ & $-0,29 * *$ & $0,34^{* *}$ & $0,56 * *$ & $0,19^{*}$ & 1 & $-0,41^{* *}$ & $0,54 * *$ \\
\hline F actor IV & $0,61 * *$ & $0,47^{* *}$ & $-0,41^{* *}$ & $0,43 * *$ & $-0,41 * *$ & 0,08 & 1 & $-0,59^{* *}$ \\
\hline$F$ actor $V$ & $-0,58 * *$ & $-0,48 * *$ & $0,31^{* *}$ & $0,50 * *$ & $0,26 * *$ & $0,54 * *$ & $-0,59 * *$ & 1 \\
\hline
\end{tabular}

**. La correlación es significativa al nivel 0,01 (bilateral).

*. La correlación es significativa al nivel 0,05 (bilateral). 


\section{Discusión}

La tasa de respuesta obtenida supuso ya una actitud que reflejó temor o falta de interés para evidenciar cuestiones relacionadas con el burnout en este colectivo y, a su vez, pudo ser la razón por la que se evidenció bajo número de casos de burnout confirmados. Autores como Longas et al. (2012) obtuvieron resultados similares a los hallados en este estudio en una investigación con docentes en Cataluña.

Los datos de participación del profesorado nos permitieron hablar de un colectivo bastante experimentado, aspecto que dio cierta garantía de que las respuestas obtenidas no fueron fruto de la inseguridad que normalmente se asocia a la etapa inicial del ejercicio docente. En cambio, destacó la baja participación del profesorado novel. Este hecho pudo ser debido a que, en los primeros años de carrera profesional, se produce la transición de las expectativas idealistas hacia la práctica cotidiana. En este periodo, los profesores/as jóvenes, llenos de proyectos innovadores y ansiosos por llegar al centro y ponerlos en práctica, se encuentran con una realidad bien distinta. Quizás este choque entre las ilusiones forjadas y la realidad, les hace sentir inseguros y desconfiados a la hora de expresar sus opiniones y vivencias en temas relacionados con el burnout docente y deciden refugiarse en sí mismos.

La respuesta del profesorado con contrato eventual también fue baja. Lo que pudo indicar, al igual que ocurrió con el profesorado novel, temor para evidenciar cuestiones relacionadas con el burnout. En cambio, probablemente por la seguridad que aporta dicha condición administrativa, el profesorado funcionario se mostró más colaborativo para participar en investigaciones de este tipo y no le importó compartir sus vivencias acerca de un tema que, lamentablemente, afecta de lleno a la profesión docente.

Igualmente, destacó la participación mayoritaria por parte de la mujer, así como del profesorado casado y con hijos. Al respecto, Albar et al. (2004) defienden que el hecho de tener hijos/as funciona como factor de protección ante el síndrome. Dichos autores señalan que ello se relaciona con el apoyo emocional recibido por parte de la familia.

En cuanto a los niveles de resiliencia, el profesorado casado o viviendo en pareja obtuvo mayores puntuaciones en resiliencia frente al soltero. Aunque, autores como Justo (2010), confirman que el hecho de que ocurran 
problemas familiares que estén distorsionando el funcionamiento normal de la dinámica del hogar, predispone a la persona a sufrir burnout.

Asimismo, el sexo femenino de la muestra manifestó más características resilientes frente al sexo masculino. En relación a ello, Stratta et al. (2013) identificaron y compararon niveles de resiliencia cuyos resultados arrojaron diferencias significativas en las fuentes de resiliencia entre varones $y$ mujeres, siendo mayor en las mujeres, al igual que ocurrió en este estudio.

Con respecto a las dimensiones del burnout, la dimensión agotamiento emocional (burnout) obtuvo correlación directa y positiva con la dimensión cinismo (burnout) y con algunas afirmaciones pertenecientes a los factores de la escala de resiliencia que contenían aspectos negativos del trabajo. Sin embargo, la correlación fue negativa con la dimensión eficacia personal/profesional. En este contexto, la pérdida de recursos emocionales ocasionados por las demandas físicas, mentales, emocionales y relacionales del trabajo docente (el número excesivo de alumnos/as en las aulas, el exceso de tareas a realizar, las presiones temporales y la sobrecarga de trabajo, el escaso profesorado de apoyo, la inadecuación de horarios, la excesiva burocracia y el papeleo que de ello se deriva, etc,) en interacción con los recursos individuales, puede impactar negativamente en su salud y disminuir en el profesor/a los sentimientos de autoeficacia.

Los resultados hallados en el factor agotamiento emocional (burnout) coincidieron con los trabajos de López y Extremera (2017), Rodríguez et al. (2017) y Burgos et al. (2018), entre otros autores, quienes señalan que cuando en el trabajo existen altas demandas y pobres recursos, ello dificulta la consecución de objetivos, disminuyen en el profesor los sentimientos de autoeficacia y, con el tiempo, dan lugar al síndrome de quemarse por el trabajo o burnout; esto es, agotamiento, cinismo y despersonalización e ineficacia profesional. En la misma línea, Vélez et al. (2017) y Dios et al. (2018) constatan que, a medida que baja la autoeficacia, aumentan los sentimientos de agotamiento emocional y cinismo que llevan al burnout. De modo que una autopercepción de baja eficacia, como la encontrada en este componente, es un desencadenante del síndrome (Peña et al., 2018).

La dimensión cinismo (burnout) obtuvo correlación directa y positiva con la dimensión agotamiento emocional (burnout) y con afirmaciones pertenecientes a los factores de la escala de resiliencia que contenían aspectos negativos del trabajo. Por el contrario, correlacionó negativamente 


\section{Vicente de Vera \& Gabari Gambarte - Burnout y Resiliencia}

con algunos juicios de la dimensión eficacia personal/profesional.

Diferentes autores, como Llull et al. (2015) subrayan que la mayoría de las investigaciones sobre el burnout señalan al profesorado de Secundaria como el más afectado, sobre todo en cuanto a la despersonalización y a la realización personal. Explican, que las interacciones negativas con los alumnos (fricciones, conflictos y disputas) se relacionan, por un lado, de forma positiva con el agotamiento emocional y la despersonalización y, por el otro, negativamente con la baja realización personal. La misma opinión manifiestan Carlotto y Câmara (2017) y Vicente de Vera y Gabari (2019), quienes reportan que los trabajadores y trabajadoras con mayor cansancio emocional son los que sienten mayor despersonalización y menor realización personal.

En cuanto a la dimensión eficacia personal/profesional (burnout), obtuvo correlación directa con los aspectos positivos del trabajo contenidos en la escala de la resiliencia. En cambio, la correlación fue negativa con las dimensiones agotamiento emocional (burnout) y cinismo (burnout) y con algunas cuestiones de la escala de resiliencia que contenían aspectos negativos del trabajo. En relación a las valoraciones obtenidas en esta dimensión, Tejedor y Mangas (2016) explican que la percepción que tiene el sujeto de sentirse eficaz con las tareas que realiza juega un papel relevante en el desarrollo del síndrome.

Asimismo, los resultados corroboraron los trabajos de Vallejo (2017), quien defiende que la autoeficacia actúa como protector frente al burnout. De modo que, los niveles de autoeficacia tan positivos hallados en este factor, protegen al grupo de docentes de esta investigación frente al burnout. En suma, tal como refieren Lozano y Reyes (2017), cuando se posee una fuerte sensación de eficacia, se potencia el control sobre las tareas a realizar y este control ayuda a percibir el trabajo como retador, lleno de significado y propósito; a sentir satisfacción al realizar las tareas, motivación por hacer un buen trabajo.

Además, como ha quedado descrito previamente, la dimensión eficacia personal/profesional correlacionó positivamente con los aspectos positivos de la escala de la resiliencia. Coincidiendo con las apreciaciones de Bonanno (2004) y Cruz y Puentes (2017), entre otros, la capacidad de afrontamiento a los estresores expresada por el profesorado encuestado es otro de los aspectos que salvaguarda a este colectivo del burnout. 
Respecto a las puntuaciones de los factores de la escala de resiliencia, las valoraciones reflejaron que el Factor I, llamado sensibilidad o afrontamiento emocional, obtuvo correlación directa y positiva con las dimensiones agotamiento emocional y cinismo del burnout y con algunos aspectos negativos contenidos en el Factor II (superación o afrontamiento conductual), Factor III (positivismo u optimismo ingenuo), Factor IV (pensamiento estereotipado) y Factor V (tolerancia a la frustración) de la escala de la resiliencia. Sin embargo, dicho Factor I mantuvo correlación negativa con la dimensión eficacia personal/profesional del burnout.

En relación a estos datos, Aldás (2017) también halla correlaciones significativas en sentido positivo entre el cansancio emocional y las estrategias de evitación y autofocalización negativa. Por su parte, Félix Verduzco et al. (2018) puntualizan que estrategias inadecuadas, de tipo escapista, aumentan la probabilidad de sufrir burnout, tal como indican las correlaciones del Factor I que acabamos de exponer. De manera que, cuando el individuo emplea estrategias de afrontamiento que no son acertadas para hacer frente a una determinada situación, crece la posibilidad de padecer burnout.

El Factor II, denominado superación o afrontamiento conductual, como explican Lázarus y Folkman (1986), mide la tendencia a pensar automáticamente en términos que faciliten una acción eficaz. Los resultados presentaron correlación positiva con la dimensión eficacia personal/profesional. Dicho Factor II también mostró correlación positiva con el Factor II (superación o afrontamiento conductual), Factor III (positivismo u optimismo ingenuo), Factor IV (pensamiento estereotipado) y Factor V (tolerancia a la frustración) de la escala de la resiliencia. En cambio, la correlación fue negativa con las dimensiones agotamiento emocional y cinismo de la escala del burnout.

Haciendo referencia a los aspectos evaluados en este factor, Ornelas (2016), González et al. (2017) y Alarcón (2018) subrayan que algunas personas son más resistentes a los estresores, lo que les posibilita poner en marcha estrategias de afrontamiento para superar las demandas que les genera estrés y les permite lograr una respuesta eficaz para restablecer el equilibrio de la situación. Del mismo modo, Medrano (2017) y Noreña (2018), entre otros autores, confirman que el uso de estrategias de afrontamiento constructivas centradas en el problema, como las encontradas 


\section{Vicente de Vera \& Gabari Gambarte-Burnout y Resiliencia}

en este componente, previene el desarrollo del síndrome.

En cuanto al Factor III, referido al positivismo u optimismo ingenuo del profesorado de este estudio, obtuvo correlación directa y positiva con la dimensión eficacia personal/profesional y con el Factor I (sensibilidad o afrontamiento emocional), Factor II (superación o afrontamiento conductual) y Factor V (tolerancia a la frustración). Por el contrario, dicho Factor III correlacionó negativamente con las dimensiones agotamiento emocional y cinismo del burnout y con el Factor IV (pensamiento estereotipado) de la escala de la resiliencia.

Los resultados de este componente coincidieron con las investigaciones de McKergow (2009), quien postula que las emociones positivas provocan, fundamentalmente, cambios en la actividad cognitiva y posteriormente cambios en la esfera conductual. Esto favorece la construcción de recursos personales (físicos, psicológicos y sociales) para afrontar las situaciones difíciles o problemáticas, que propicia un estilo de afrontamiento adaptativo o resiliente. Es decir, la experiencia de emociones positivas no es más que el reflejo de un modo resiliente de afrontar las situaciones adversas (Oriol et al., 2017). Asimismo, en relación a los datos hallados en este factor, Pulido y Herrera (2018) corroboran que el optimismo ejerce una influencia diferencial en la valoración y afrontamiento de las dificultades, en el desenvolvimiento en el mundo social y académico y en el bienestar psíquico $\mathrm{y}$ físico de las personas $\mathrm{y}$, señalan, que optimismo y pesimismo correlacionan de manera significativa con la autoeficacia y con desgaste profesional de manera diversa.

Tras el análisis del Factor IV o pensamiento estereotipado, entendido como la tendencia de algunas personas a dejarse llevar por las ideas establecidas socialmente, correlacionó directa y positivamente con las dimensiones agotamiento emocional y cinismo del burnout y con algunos aspectos negativos del trabajo contenidos en el Factor I (sensibilidad o afrontamiento emocional). En cambio, la correlación de dicho Factor IV fue negativa con la dimensión eficacia personal/profesional, con el Factor II (superación o afrontamiento conductual) y con el Factor V (tolerancia a la frustración) de la escala de resiliencia.

Según hemos expuesto, el pensamiento estereotipado está relacionado con un rasgo de la personalidad denominado locus de control. Este concepto hace alusión al control que el sujeto se atribuye sobre sus actos. De modo 
que, los docentes con locus de control interno, es decir, que creen que los sucesos que ocurren en su ambiente son consecuencia de sus conductas y, por tanto, controlables, evalúan la enseñanza como menos estresante (Esteras et al., 2016; Torres y Bonilla, 2017). En sentido contrario, en relación a las valoraciones de este factor $\mathrm{y}$, de acuerdo con las aportaciones de Vargas et al. (2016), el profesorado que manifestó locus de control externo tiene más probabilidades de sufrir burnout; lo que conlleva mayor agotamiento emocional y despersonalización, así como menor logro personal. Esto es, ante situaciones ambiguas, difíciles o novedosas en las que el sujeto cree tener poca o ninguna posibilidad de controlar, existen mayores posibilidades de que el síndrome aparezca (Islas et al., 2017).

Por último, el Factor $\mathrm{V}$ se refiere a la tolerancia a la frustración del profesorado, cuya puntuación fue mayor en aquellos docentes con personalidad resistente. Los sujetos que obtuvieron alta puntuación en este componente puntuaron también alto en la dimensión eficacia personal/profesional y en el Factor I (sensibilidad afrontamiento emocional), Factor II (superación o afrontamiento conductual) y Factor III (positivismo u optimismo ingenuo). Por el contrario, la correlación fue negativa con las dimensiones agotamiento emocional y cinismo del burnout y con el Factor IV (pensamiento estereotipado) de la escala de la resiliencia. En relación a los resultados reportados, autores como González et al. (2017), Vargas et al. (2017) y Carrara (2018), informan que las personas con personalidad resistente son más eficaces para hacer frente al estrés y tienden a desgastarse menos profesionalmente, en comparación a las que no tienen este patrón de personalidad.

\section{Conclusiones}

Los resultados hallados en este estudio permiten establecer niveles de relación entre burnout y resiliencia expresados. De modo que, en presencia de situaciones de adversidad laboral, los docentes más resilientes no llegan a quemarse, sino que logran mayores habilidades y competencias de compromiso laboral, a la vez que tienen la capacidad de utilizar su energía e implicación personal para sobreponerse a las dificultades, adaptarse adecuadamente y experimentar emociones positivas. En cambio, los 


\section{Vicente de Vera \& Gabari Gambarte - Burnout y Resiliencia}

docentes menos resilientes tienen predisposición a las emociones negativas, a sobrevalorar el riesgo, e incrementar su efecto con cansancio e indiferencia en el trabajo. Se concluye, por tanto, que la resiliencia disminuye la vulnerabilidad al burnout.

Además, algunas variables (ej., el positivismo, las estrategias de afrontamiento constructivas, alta resistencia a la frustración, la autoeficacia, etc.) con los que el sujeto hace frente a las demandas laborales, actúan como factores de protección frente al burnout. Mientras que los factores de riesgo (ej., las estrategias de afrontamiento emocional, la escasa tolerancia a la frustración, el clima familiar negativo, las escasas oportunidades laborales, etc.) son un predictor importante en el desarrollo del síndrome.

Adicionalmente, una actitud optimista como la mostrada por los docentes de este estudio, se relaciona con la confianza y la persistencia en la conducta. Por el contrario, una actitud pesimista hace que las personas se manifiesten dudosas y vacilantes. Como es de suponer, estas diferencias tienen consecuencias en la forma de afrontar los cambios, la adversidad y la experiencia de estrés en el trabajo. De manera que, con una actitud optimista, se tienden a utilizar estrategias centradas en el problema, especialmente cuando la situación es controlable. Por tanto, las emociones positivas son un elemento positivo ante la adversidad y favorecedores del surgimiento de la resiliencia del colectivo encuestado. En la medida que refuerzan estos atributos, mitigan la vulnerabilidad al burnout.

Asimismo, una respuesta eficaz frente a las demandas contextuales, como la expresada por los docentes participantes, está relacionada de manera directa con la autoeficacia, entendida como aquella sensación interna que la persona experimenta, que le lleva a sentir y a pensar que es competente y le posibilita creer en sus propias capacidades. Estos juicios de autoeficacia influyen en las metas que las personas se marcan y en sus respuestas afectivas ante los niveles de logro alcanzados. Se concluye, que algunas variables de la personalidad, como la resiliencia y la autoeficacia, protegen al profesorado de este estudio frente al síndrome de burnout.

Consideramos que los hallazgos en este estudio con respecto al síndrome de burnout y la resiliencia necesitan en el futuro del diseño de investigaciones adecuadas para conocer con más detalle las implicaciones que ambos constructos tienen en el trabajo de estos profesionales. Desde estas líneas, hacemos hincapié en la importancia que adquiere el desarrollo 
de la resiliencia en el desempeño docente para afrontar las demandas laborales, ya que actúa como amortiguador de los estresores y disminuye la vulnerabilidad al burnout. Incidimos igualmente en la influencia determinante que la autoeficacia tiene en el rendimiento exitoso, en la motivación y en la persistencia de estos profesionales.

Por otro lado, en esta investigación nos hemos centrado en el profesorado de Educación Secundaria, aspecto que puede interpretarse como una limitación a la hora de generalizar los resultados al colectivo docente. Abrir el abanico a todos los niveles y etapas puede ser excelente, pero también muy complejo por la variedad intrínseca que esto conlleva. Asimismo, nos parece interesante utilizar un diseño longitudinal con otros momentos e instrumentos de recogida de datos, así como realizar estudios comparativos en otras provincias/comunidades autónomas. No obstante, a pesar de las limitaciones comentadas, creemos que los resultados presentados en este estudio pueden tener importantes repercusiones prácticas de cara a facilitar el bienestar del profesorado de Secundaria.

\section{Referencias}

Alarcón, M. E. B. (2018). Estrés y Burnout Enfermedades En La Vida Actual. Palibrio.

Albar, M. J., Romero, M. E., González, M. D., Carbayo, E., García, A., Gutiérrez, I. \& Algaba, S. (2004). Apoyo social, características sociodemográficas y burnout en enfermeras y auxiliares del hospital. Enfermería Clínica, 14(5), 281-285. doi: 10.1016/S11308621(04)73899-1

Aldás, A. C. (2017). Estrategias de afrontamiento y sindrome de burnout en personal de salud (thesis, Universidad Técnica de Ambato-Facultad de Ciencias de la Salud-Carrera de Psicología Clínica). Recuperado de:http://repo.uta.edu.ec/bitstream/123456789/26500/2/Ana\%20Caroli na\%20Ald\%C3\%A1s\%20Villac\%C3\%ADs\%20pdf.pdf

Bonanno, G. A. (2004). Loss, trauma, and human resilience: have we underestimated the human capacity to thrive after extremely aversive events? American psychologist, 59(1), 20-28. doi: 10.1037/0003066X.59.1.20

Bresó, E., Salanova, M., Schaufeli, W. B \& Nogareda, C. (2007). NTP N 732. Sindrome de estar quemado por el trabajo Burnout (III): 
Instrumento de medición. Madrid: Instituto Nacional de Seguridad e Higiene en el Trabajo, Ministerio de Trabajo y Asuntos Sociales.

Burgos, A. L. V., Paris, A. P. D., Salcedo, A. G. S. \& Arriagada, A. A. (2018). Psychological well-being and burnout in primary health care professionals in the region of Los Lagos, Chile. Acta Universitaria, 28(3), 56-64.

Carlotto, M. S. \& Câmara, S. G. (2017). Psychosocial Risks Associated with Burnout Syndrome Among University Professors. Avances en Psicología Latinoamericana, 35(3), 447-457. doi: 10.12804/revistas.urosario.edu.co/apl/a.4036

Carrara, J. L. (2018). Personalidad resistente y su relación con el egreso voluntario en call centers. Revista de Psicología, 36(1), 239-263. doi: 10.18800/psico.201801.009

Cruz, D. M. \& Puentes, A. (2017). Relationship between different dimensions of the Burnout syndrome and the coping strategies implemented by security guards at a private corporation from Tunja (Col). Psicogente, 20(38), 268-281.

Dios, I., Calmaestra, J. \& Rodríguez, A. J. (2018). Validación de la escala de competencias docentes organizacionales y didácticas para educadores. Revista mexicana de investigación educativa, 23(76), 281-302.

Esteras, J., Sandín, B. \& Chorot, P. (2016). El síndrome de burnout en los docentes: Los efectos de las variables psicosociales. In Castejón Costa, Juan Luis (coord.). Psicología y educación: Presente y futuro (pp. 1177-1185). Alicante: ACIPE. ISBN 978-84-608-8714-0

Félix, R. O., García, C. \& Mercado, S. M. (2018). El estrés en el entorno laboral. Revisión genérica desde la teoría. CULCyT, (64), 31-42.

González, H., Pelegrín, A. \& Carballo, J. (2017). Ira y personalidad resistente en deportistas de raqueta y resistencia. Revista Iberoamericana de Ciencias de la Actividad Física y el Deporte, 6(2), 21-29. doi: 10.24310/riccafd.2017.v6i2.3776

González, T. M. P., Torres, L. H. \& Carreres, A. L. (2017). Fortalezas y virtudes personales del profesorado y su relación con la eficacia docente. International Journal of Developmental and Educational Psychology (Revista INFAD de Psicología), 7(1), 141-150. doi: 10.17060/ijodaep.2014.n1.v7.785

Islas, R. A. C., Gutiérrez, F. J. C., Castellanos, J. L. \& Méndez, K. P. (2017). 
Síndrome de burnout en médicos docentes de un hospital de $2^{\circ}$ nivel en México. Educación Médica, 18(4), 254-261. doi: 10.1016/j.edumed.2016.09.001

Justo, C. F. (2010). Intervención sobre los niveles de burnout y resiliencia en docentes de educación secundaria a través de un programa de conciencia plena (mindfulness) Revista Complutense de Educación, 21(2), 271-288.

Lazarus, R. S. \& Folkman, S. (1986). Cognitive theories and the issue of circularity. In M. H. Appley \& R. Trumbull (Eds.), Dynamics of stress, (pp.63-80). New York: Plenum. doi: 10.1007/978-1-46845122-1 4

Llull, D. S., Cerdà, M. X. M. \& Brage, L. B. (2015). Malestar social y malestar docente: una investigación sobre el síndrome de desgaste profesional burnout y su incidencia socioeducativa. Aula: Revista de Pedagogía de la Universidad de Salamanca, 21(1), 245-257. doi: 10.14201/aula201521245257

Longas, J., Chamarro, A., Riera, J. \& Cladellas, R. (2012). La incidencia del contexto interno docente en la aparición del Síndrome del Quemado por el trabajo en profesionales de la enseñanza. Revista de Psicología del Trabajo y de las Organizaciones, 28(2), 107-118. doi: 10.5093/tr2012a9

López, S. M. \& Extremera, N. (2017). Estado de la cuestión sobre inteligencia emocional y burnout en el profesorado por países, año de publicación, ciclos educativos e instrumentos de evaluación. Profesorado, Revista de Currículum y Formación del Profesorado, 21(3), 371-389.

Lozano, C. R. \& Reyes, M. (2017). Docentes universitarios: Una mirada desde la Autoeficacia general y engagement laboral. Revista Digital de Investigación en Docencia Universitaria, 11(1), 134-148. doi: 10.19083/ridu.11.503

Martínez, S. M. (2010). Construcción y análisis psicométrico de un Cuestionario de evaluación de la resiliencia. Recuperado de http://www. unizar. es/abarrasa/tea/200910_25906/serrano2010.pdf

McKergow, M. (2009). Positivity: Groundbreaking research reveals how to embrace the hidden strengths of positive emotions, overcome negativity and thrive. InterAction-The Journal of Solution Focus in 
Organisations, 1(1), 89-92.

Medrano, F. M. (2017). Estilos de afrontamiento del estrés que aplica el profesional de enfermería en el servicio de emergencia del Hospital Nacional Daniel Alcides Carrión mayo-2016. Recuperado de http://repositorio.upsjb.edu.pe/handle/upsjb/1368

Noreña, H. (2018). Estrés académico y estrategias de afrontamiento en estudiantes de primer, segundo y tercer semestre de la Facultad de Ingeniería de la Universidad de Antioquia, 2017 (Doctoral dissertation, Psicologia). Recuperado de http://200.24.17.74:8080/jspui/bitstream/fcsh/1138/1/Nore\%C3\%B1a Henry_2018_Estr\%C3\%A9sAcad\%C3\%A9micoEstrategias\%20.pdf

Oriol, X., Mendoza, M., Covarrubias, C. G. \& Molina, V. M. (2017). Emociones positivas, apoyo a la autonomía y rendimiento de estudiantes universitarios: el papel mediador del compromiso académico y la autoeficacia. Revista de Psicodidáctica, 22(1), 45-53. doi: 10.1016/S1136-1034(17)30043-6

Ornelas, P. E. (2016). Estrategias de afrontamiento y resiliencia en cuidadores primarios con duelo. Revista Psicología y Salud, 26(2), 177-184.

Peña, J. E., Raso, P. C. \& Ferrero, B. S. (2018). El síndrome de burnout en los docentes. Madrid: Ediciones Pirámide.

Pulido, F. \& Herrera, F. (2018). Predictors of Happiness and Emotional Intelligence in Secondary Education. Revista Colombiana de Psicología, 27(1), 71-84. doi: 10.15446/rcp.v27n1.62705

Rodríguez, A. M., Sola, T. \& Fernández, M. (2017). Impacto del Burnout en el desarrollo profesional del profesorado universitario. Una revisión de la investigación. Revista Electrónica Interuniversitaria de Formación del Profesorado, 20(3), 161-178. doi: 10.6018/reifop.20.3.275121

Salanova, M., Schaufeli, W. B., Llorens, S., Peiró, J. M. \& Grau, R. (2000). Desde el burnout al engagement: ¿una nueva perspectiva? Revista de Psicología del Trabajo y las Organizaciones, 16(1), 117-134.

Schaufeli W. B., Leiter M. P., Maslach, C. \& Jackson S. E. (1996). The Maslach Burnout Inventory: General Survey (MBI-GS). In C. Maslach, S., Jackson \& M. Leiter (eds.): Maslach Burnout Inventory Manual, $3^{\text {a }}$ ed. (pp. 19-26). Palo Alto, California: Consulting 
Psychologists Press.

Stratta, P., Capanna, C., Patriarca, S., de Cataldo, S., Bonanni, R. L., Riccardi, I. \& Rossi, A. (2013). Resilience in adolescence: gender differences two years after the earthquake of L'Aquila. Personality and Individual Differences, 54(3), 327-331. doi: 10.1016/j.paid.2012.09.016

Tejedor, E. M. \& Mangas, S. L. (2016). La autoeficacia y la autorregulación como variables moderadoras del estrés laboral en docentes de Educación Primaria. Universitas Psychologica, 15(1), 15-28.

Torres, D. \& Bonilla, L. R. (2017). Burnout académico en estudiantes de psicología. Psicolnnova, 1(1), 1-15.

Vallejo, M. (2017). Una aproximación al síndrome de burnout y las características laborales de emigrantes españoles en países europeos. Revista de Psicología del Trabajo y de las Organizaciones, 33(2), 137-145. doi: 10.1016/j.rpto.2017.02.001

Vargas, J. D. P., Briñez, V. S., Segura, M. C. N. \& Nieto, A. A. (2016). Creencias irracionales, síndrome de Burnout y adicción al trabajo en las organizaciones. Psicogente, 19(35), 148-160.

Vargas, L. D., Niño, C. L. \& Acosta, J. Y. (2017). Estrategias que modulan el síndrome de Burnout en enfermeros (as): una revisión bibliográfica. Revista Ciencia y Cuidado, 14(1), 111-131. doi: 10.22463/17949831.810

Vélez, A. P., López, J. J. \& González, J. B. (2017). Habilidades emocionales y profesionalización docente para la educación inclusiva en la sociedad en red. Contextos Educativos. Revista de Educación, 20(1), 201-215. doi: 10.18172/con.3011

Vicente de Vera, M. I. \& Gabari, M. I. (2019). Liderazgo Pedagógico en Educación Secundaria: Aportaciones desde la Evaluación de BurnoutResiliencia en Docentes. International Journal of Educational Leadership and Management, 7(1), 104-134. doi: 10.17583/ijelm.2018.3519 
152 Vicente de Vera \& Gabari Gambarte - Burnout y Resiliencia

M. Inmaculada Vicente de Vera is professor at the Public University of Navarra, Spain

M. Inés Gabari Gambarte is professor at the Public University of Navarra, Spain

Contact Address: ivicentedevera@yahoo.es 